\title{
Montecarlo Simulation Applied to Measurement of the Impact of the Smart Antenna Technology in Digital Cellular Systems
}

\author{
Simulación montecarlo aplicada a la medición del impacto de la tecnología de \\ antenas inteligentes en sistemas celulares digitales
}

\author{
Castañeda-Camacho Josefina \\ Electronic Department \\ Benemérita Universidad Autónoma de Puebla \\ E-mail:jcastane@ece.buap.mx \\ Mino-Aguilar Gerardo \\ Electronic Department \\ Benemérita Universidad Autónoma de Puebla \\ E-mail:gmino44@ieee.org \\ Cortez Liliana \\ Electronic Department \\ Benemérita Universidad Autónoma de Puebla \\ E-mail:1cortez@ece.buap.mx
}

\author{
Gutiérrez-Arias José Eligio Moisés \\ Electronic Department \\ Benemérita Universidad Autónoma de Puebla \\ E-mail:jmgutierrez@ece.buap.mx \\ Guerrero-Castellanos José Fermi \\ Electronic Department \\ Benemérita Universidad Autónoma de Puebla \\ E-mail:fguerrero@ece.buap.mx \\ Muñóz-Hernández Germán Ardul \\ Electronic Department \\ Benemérita Universidad Autónoma de Puebla \\ E-mail:gmunoz64@yahoo.co.uk
}

Information on the article: received: October 2013, reevaluated: January 2014, accepted: May 2014

\begin{abstract}
The smart antenna technology has received increasing interest due to its capability for improving the performance of wireless radio systems. In this work, we studied the throughput maximization in a digital cellular system when a smart antenna array is implemented. We focus, in the study of the downlink of a $3 \mathrm{G}$ cellular system and consider a packet data direct-sequence code division, multiple access (DS-CDMA). Our methodology is based on the Montecarlo simulation technique, and it is used to show that it is possible to obtain a significant increment in the throughput of the system due to the switched beam smart antenna array. From our results we conclude that it is feasible to consider the application of this technology in 4G environments.
\end{abstract}

\section{Keywords:}

- smart antenna

- Montecarlo simulation and

- Digital Cellular System 


\section{Resumen}

La tecnología de antenas inteligentes ha sido de gran interés debido a su capacidad para mejorar el desempeño de los sistemas inalámbricos. En este trabajo, estudiamos la mejora del desempeño en el sistema digital celular cuando se utiliza un arreglo de antenas inteligentes. Nos enfocamos además, en el estudio del enlace de bajada de un sistema celular 3G y consideramos un esquema de acceso múltiple de paquetes de datos de secuencia directa (DS-CDMA). Nuestra metodología está basada en la técnica de simulación Montecarlo y se usa para mostrar que es posible obtener un incremento significativo en el desempeño del sistema debido al arreglo de antenas inteligentes. De nuestros resultados concluimos que es posible considerar la aplicación de esta tecnología en ambientes 4G.

\section{Introduction}

The adoption of smart antenna techniques in future wireless systems is expected to have a significant impact on the efficient use of the spectrum, the minimization of the cost of establishing new wireless networks, the optimization of service quality, and realization of transparent operation across wireless networks. Adaptive antennas have been used for decades in areas such as radars, satellite communications, remote sensing, and direction finding, to name a few. Each of these applications is associated with its own unique set of challenges, such as the channel in which the system operates, the propagation environment, sources of interference, and noise or jamming. In addition, the end goal for which the adaptive antenna is used affects the selection of the type of array, size, adaptive algorithms, and integration with other system components (Alexiou and Haardt, 2004; Liberti and Rapaport, 1999).

Current mobile communications systems employ sectorization to reduce interference and increase capacity. Cells are broken into three or six sectors, with dedicated antennas and RF paths. Increasing the amount of sectorization reduces the interference seen by the desired signal. One drawback of current sectorization techniques is that its efficiency decreases as the number of sectors increase, due to the antenna pattern overlap. Furthermore, increasing the number of sectors increases the handoffs in the mobile experiences while moving across the cell. Smart antennas offer a more efficient way to reduce interference in mobile communications systems, through the use of narrow beams directed toward clusters of users, while at the same time steering nulls toward interfering users. With a narrow-beam directed toward a desired user, some interferers that would have seen in the $120^{\circ}$ sector antenna, will be outside of the beam-width of the array (Herscovici, 2001).
Achieving peak data rates specified in each standard in a real system remains very unlikely because it would require an unloaded system serving a single user to be extremely close to the base station. This leads to two questions: Why the increased interest in the smart antennas?, and how are they being considered as a viable technology for applications such as mobile telecommunications? As we know, operators are faced with increasing capacity demands for both voice and data services. Although various $3 G$ technologies offer higher data rates and double voice capacity compared with their $2 \mathrm{G}$ counterparts, their actual performance is still susceptible to interference, and adverse channel conditions created by multipath propagation and system loading. As such, smart antennas techniques can complement $3 \mathrm{G}$ system and improve their performance by alleviating and reducing the degradation caused by the aforementioned factors. In fact, because of their nature, technologies such as HSDPA and 1xEV-DO can greatly benefit from smart antennas since any improvement in the SIR experienced by the users would directly translate to better throughput for individual users as well as increased sector throughput that can support higher capacities (Liberti and Rapaport, 1999; Herscovici, 2001; Bender et al., 2000) A new generation of mobile technology is marked by a significant advance in functionality (Etoh, 2005; Yavuz et al., 2006). Smart antennas techniques can complement the new generation systems by improving their performance.

In this work we analyze the benefit of the smart antennas in CDMA2000 1xEV-DO systems in terms of the throughput of the data network or the maximum achievable data transmission rate; in a sense that any improvement in the SIR experienced by the users would directly translate to better throughput for individual users as well as increased sector throughput that can support higher capacities (Herscovici, 2001; Bender et al., 2000). 
We evaluate the throughput in the forward link of a system configuration which consists of 37 macrocells. The base stations are located in the center position and the use of three sectored antennas is assumed. The mobile users are uniformly distributed within the cells. The performance of the system has been measured in terms of a Monte Carlo simulation implemented in MATLAB. The Monte Carlo simulation methodology is a type of simulation that relies on repeated random sampling and statistical analysis to compute the results (Raychaudhuri, 2008; Zhuang and Lin, 2007). This method of simulation is very closely related to random experiments, experiments for which the specific result is not known in advance. In this context, Monte Carlo simulation can be considered as a methodical way of doing so-called what-if analysis where we use mathematical models to describe the interactions in a system using mathematical expressions. These models typically depend on a number of input parameters, which when processed through the mathematical formulas in the model, results in one or more outputs.

This work is organized as follows. In the next section, we present the operation of the CDMA2000 $1 \times$ EV-DO system. In the third section we evaluate the performance of the CDMA2000 1xEV-DO system. The numerical results are presented in the fourth section. The last section provides our conclusions and remarks.

\section{CDMA2000 1xEV-DO System}

As we can see in Table 1, different technologies support different peak data rates. The peak rate is the maximum transmission speed which an individual user may experience under ideal conditions (i.e., it only affects the user experience). Data throughput on the other hand, is a far more important metric for performance. Thus, the user throughput is the average data rate a user may experience. Table I compares different peak data rates and throughput for different 3G technologies (Liberti and Rapaport, 1999).

The CDMA2000 family of standards is a wideband spread spectrum radio interface that uses CDMA technology to meet the objectives of $3 G$ systems while maintaining backward compatibility with IS-95 based systems. This means that mobile handsets designed according to the IS-95 standard are capable to operate in a CDMA2000 system and vice versa. The first component of the CDMA2000 standard is called 1x radio transmission technology (1xRTT) because it uses an RF carrier of 1.25 MHz just like IS-95 based systems, hence the $1 \mathrm{x}$, is also referred to as spreading rate (SR) 1 . The key bene- fits of the 1xRTT technology standardized under the name of IS-2000 compared with IS-95A/B standards can be summarized as follows: Better forward error correction (FEC), fast forward link power control mechanism and multimedia services and improved data services support (Liberti and Rapaport, 1999).

The current 3G operators in Japan and Korea as well as in the United States are already experiencing great success with their data services. The 1xEV-DO standard is optimized for wireless high speed packet data services (Liberti and Rapaport, 1999; Esteves et al., 2003; Wonsuk et al., 2001);. Because of the typical asymmetric characteristics of the IP traffic, the downlink is the most critical of the two links (Bender et al., 2000; Esteves et al., 2003). Thus several techniques were introduced in 1xEV-DO to optimize the downlink throughput. Timedivision-multiplexed (TDM) waveform, power control and a variety of modulation schemes, including QPSK, 8 PSK and 16 QAM as well as coding rates that best match the fading channel, are defined in what is commonly called adaptive modulation and coding techniques (Yavuz and Paranchych, 2003; Yonghoon and Youngnam, 2002)

\section{Forward Link Performance.}

In the CDMA2000 1xEV-DO system when the transmission rate of user $i$ is $R_{i}$ and the bandwidth is given by $W_{C}$ the energy to interference ratio requirement is given by

$$
\gamma=\left(\frac{E_{b}}{I_{0}}\right)_{i}=\frac{W_{C}}{R_{i}} S I R_{i}
$$

where $S I R_{i}$ is the signal to interference ratio requirement. If we consider QPSK modulation and the transmission rate as a function of the processing gain, the maximum achievable transmission rate for the user $i$, which we define as throughput, can be written as (Yonghoon and Youngnam, 2002; Ulukus and Greenstain, 2000).

Table 1. 3G Technologies Comparisons

\begin{tabular}{lccc}
\hline & $\begin{array}{c}\text { Carrier } \\
\text { Bandwidth } \\
\text { /Spectrum } \\
(\mathrm{MHz})\end{array}$ & $\begin{array}{c}\text { Downlink } \\
\text { Peak Data } \\
\text { Rate(Kbps) }\end{array}$ & $\begin{array}{c}\text { Average User } \\
\text { Throughput } \\
(\text { Kbps })\end{array}$ \\
\hline CDMA20001x & $1.25 / 1.25$ & 153.6 & $60-80$ \\
CDMA2000 & $1.25 / 1.25$ & 2458 & $300-500$ \\
1xEV-DO Rev. 0 & $3.85 / 5$ & 384 & $220-320$ \\
WCDMA & $3.84-/-5$ & 14400 & $550-1100$ \\
HSDPA & & & \\
\hline
\end{tabular}




$$
R_{i}=\left\{\begin{array}{cc}
\frac{W_{C}}{\gamma} \operatorname{SIR}_{i} & S I R_{i}<2 \gamma \\
2 W_{C} & S I R_{i} \geq 2 \gamma
\end{array}\right.
$$

For the CDMA2000 1xEV-DO system, the signal to interference ratio is given by

$$
\operatorname{SIR}_{i}=\frac{G\left(\varphi_{0, i}\right) / 10^{\varsigma_{0, i} / 10} r_{0, i}^{\mu}}{\sum_{k=1}^{N_{B S}} G\left(\varphi_{k, i}\right) / 10^{\varsigma_{k, i} / 10} r_{k, i}^{\mu}}
$$

where $G\left(\varphi_{0,}\right)$ is the antenna gain relative to the $i$-th user respect to the 0 -th base station, $G\left(\varphi_{k^{\prime}}\right)$ is the antenna gain relative to the $i$-th user with respect to the $k$-th base station $10^{\varsigma_{k, i} / 10} r_{k, i}^{\mu}$ models the propagation losses given by the product of the $\mu$-th power of the $r_{k, i}$ distance between the CDMA2000 1xEV-DO interest user in the 0 -th base station and the $k$-th base station, and a lognormal component which represents the shadowing losses and whose characteristic Gaussian random variable, $\zeta_{k, i}$ has zero mean and standard deviation $\Delta$. The $10^{\xi_{k, j} / 10} r_{k, j}^{\mu}$ models the propagation losses given by the product of the $\mu$-th power of the $r_{k, j}$ distance between the $j$-th user and its $k$-th power control base station and a lognormal component which represents the shadowing losses. $N_{B S}$ is the number of interfering base stations.

In the previous equation the antenna gain is approximated by a parabolic function in the main beam and by a constant average for the side-lobe. Therefore, the antenna gain can be expressed as (Ramakrishna and Holtzman, 1998; Castañeda y Lara, 2008).

$$
G\left(\phi_{k, j}\right)=\left\{\begin{array}{cl}
1-\frac{1-q}{\theta^{2}} \phi_{k, j}^{2} & \left|\phi_{k, j}\right| \leq \sqrt{\frac{1-p}{1-q}} \theta \\
p & \left|\phi_{k, j}\right|>\sqrt{\frac{1-p}{1-q}} \theta
\end{array}\right.
$$

where $\phi$ is the angle between the desired user and the direction of the maximum gain of the antenna, $q$ represents the antenna gain level (normalized to the maximum gain) at different $\theta$ sector crossover from the maximum gain direction and $p$ represents the average normalized gain level for the side-lobe. The nominal values chosen in our evaluations for $q$ and $p$ were -4 and $-15 \mathrm{~dB}$ respectively. This corresponds to a typical cellular sector antenna with 3-dB horizontal beam width around $100-110^{\circ}$.

\section{Numerical results}

A Monte Carlo simulation of discrete events was carried out in a cellular CDMA2000 1xEV-DO system. We evaluated the performance in terms of the throughput given by the equation (2), where the SIR is calculated in the scenario proposed in Figure 1. In addition we

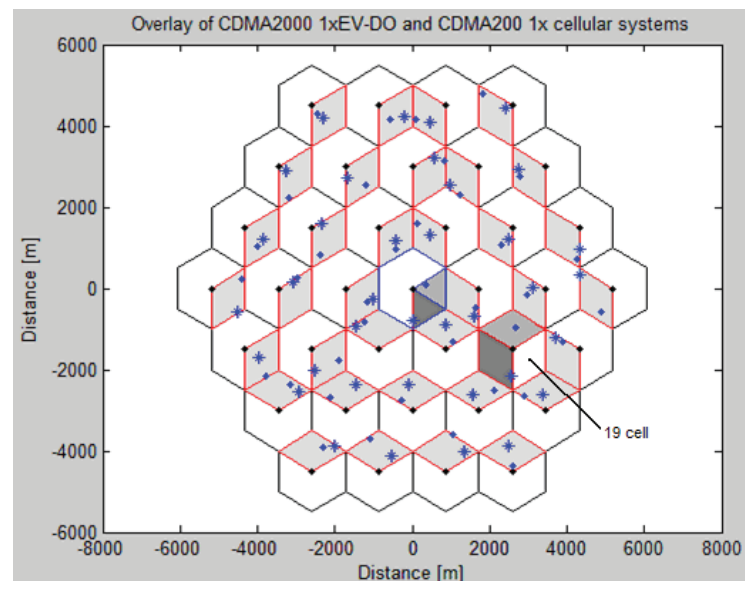

Figure 1. Macrocell CDMA1xEV-DO system scenario

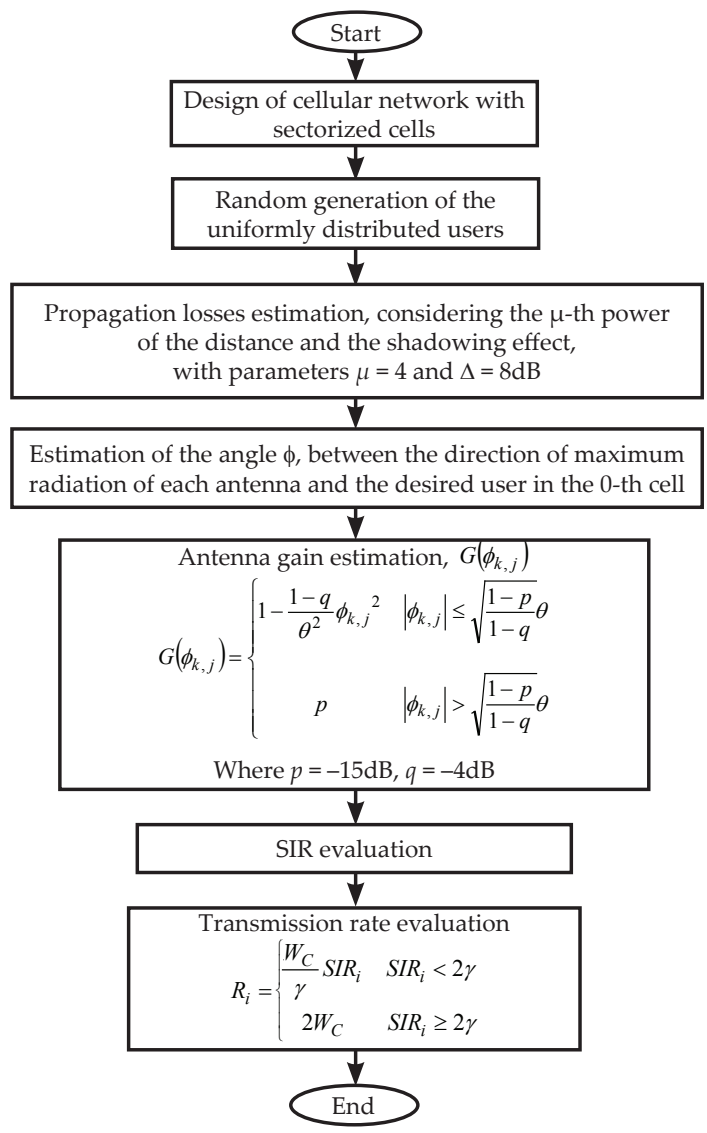

Figure 2. Dataflow of the Monte Carlo simulation 


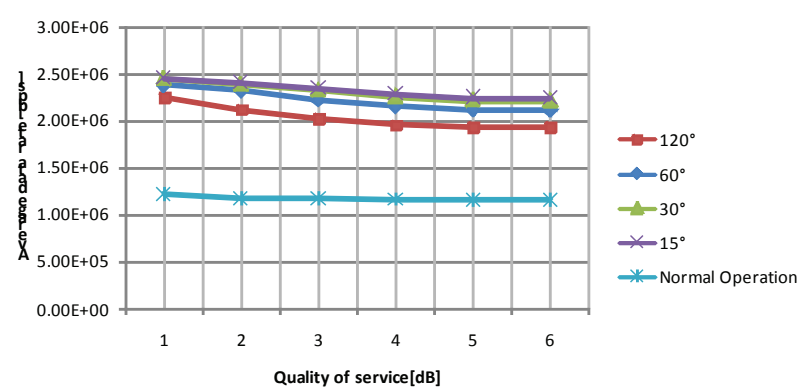

Figure 3. Throughput [bps] of the CDMA1xEV-DO system

assumed that $\Delta=8 \mathrm{~dB}, \mu=4, W_{\mathrm{C}}=1.2288 \mathrm{MHz}$ and the cell size of $1 \mathrm{Km}$. In equation (3) we are considering that $N_{B S}=36$ in a 37-cell system with one center cell and 36 interfering cells as we can see in Figure 1.

The development of the Monte Carlo simulation can be summarized by the dataflow in Figure 2 .

Finally, Figure 3 shows the average data rate or maximum achievable data transmission rate of the CDMA2000 1xEV-DO system for different $\gamma$ values and for different, $\theta$, beam-width values.

Compared with the normal operation, in which the CDMA2000 1xEV-DO system does not implement smart antennas, we can observe that the throughput increases as the beam width of the antenna array decreases. From the results, when we consider for example a $\theta=15^{\circ}$ we obtain a significantly improved data rate, approximately equal to $100 \%$ in comparison with the normal operation.

\section{Conclusion}

In this paper we developed a Monte Carlo simulation of discrete events to prove that it is possible to obtain a significant increment in the throughput of the 3G system due to the smart antenna array. Also, we can apply this technology to $4 \mathrm{G}$ generation systems to improve its performance. Smart antenna arrays provide an efficient mechanism to maximize the throughput of a CDMA2000 $1 \mathrm{xEV}-\mathrm{DO}$ system. In fact, results prove that the use of smart antennas in a CDMA cellular system can provide a $100 \%$ of data rate increment compared with the normal operation of the system. We are also leaving the basis for the analysis of this smart antenna technology in future wireless generations.

\section{References}

Alexiou A. and Haardt M. Smart antenna technologies for future wireless systems: Trends and Challenges. IEEE Communications Magazine, volume 42 (issue 9), 2004: 90-97.
Bender P., Black P., Grob M., Padovani R., Sindhushyana N., Viterbi A. CDMA/HDR: A bandwidth-efficient high-speed wireless data service for nomadic users. IEEE Communications Magazine, volume 38 (issue 7), July 2000: 70-77.

Castañeda-Camacho J., Uc-Rios C.E., Lara-Rodríguez D. Reverse link erlang capacity of multiclass cdma cellular system considering nonideal antenna sectorization. IEEE Transactions on Vehicular Technology, volume 52 (issue 6), November 2003: 1476-1488.

Castañeda-Camacho J. and Lara-Rodríguez D. Teletraffic analysis of an overlaid cdma and tdma systems with cell coverage area restriction. IEEE Transactions on Vehicular Technology, volume 57, (issue 2), March 2008: 828-845.

Esteves E., Gurelli M., Fan M. Performance of fixed wireless access with CDMA2000 1xEV-DO, in: IEEE 58th Vehicular Technology Conference (VTC 2003-Fall), 2003, pp. 836-840.

Etoh M. Next generation mobile systems $3 \mathrm{G}$ and beyond, John Wiley and Sons, Wiley Online Library, 2005.

Herscovici N. and Christodoulou C. Potentials of smart antennas in CDMA systems and uplink improvements. IEEE Antennas and Propagation Magazine, volume 43 (issue 5), October 2001: 172-177.

Liberti J.C. and Rapaport T.S. Smart antennas for wireless communications, Prentice Hall, 1999.

Zhuang M. and Lin B. Performance analysis of receive antennas array egc diversity with monte-carlo simulation method, IEEE International Workshop on Anti-counterfeiting, Security and Identification, IEEEXplore Digital Library, 2007, pp. 344-349.

Ramakrishna S. and Holtzman J.M. A scheme for throughput maximization in a dual class CDMA system. IEEE Journal on Selected Areas in Communication, volume 16 (issue 6), August 1998: 830-844.

Raychaudhuri S. Introduction to monte carlo simulation, in: IEEE Proceedings of the 2008 Winter Simulation Conference, 2008, pp. 91-100.

Sennor U. and Greenstain L.J. Throughput maximization in CDMA uplinks using adaptive spreading and power control, in: IEEE sixth International Symp on Spread Spectrum Techiques and Applications, volume 2, September 2000, pp. 565-569.

Sindhushayana N.T. and Black P.J. Forward link coding and modulation for CDMA2000 1xEV-DO (IS-856), in: IEEE Intl. Symp. On Personal Indoor and Mobile Radio Communications, volume 4, pp. 1839-1846, September 2002.

Wonsuk C., Hong-Woo L., Moon J. Downlink capacity of CDMA/ HDR, in: IEEE Vehicular Technology Conference (VTC 2003), Spring 2001, pp. 836-840.

Yavuz M., Díaz S., Kapoor R., Grob M., Black P., Tokgoz Y., Lott C. VoIP over cdma2000 1xEV-DO revision A. IEEE Communications Magazine, volume 44 (issue 2), February 2006, pp. 88-95.

Yavuz M. and Paranchych D.W. Adaptive rate control in high data rate wireless networks. IEEE Wireless Communications and Networking, volume 2, March 2003: 866-871.

Yonghoon C. and Youngnam H. A channel based scheeduling algorithm for CDMA2000 1xEV-DO system, in: IEEE Symp., in: Wireless Personal Multimedia Communications, volume 2, October 2002, pp. 621-625. 


\begin{abstract}
About the authors
Josefina Castañeda-Camacho (S'99). Born in Puebla, México, 1973. B.Sc. degree in electrical engineering from the Benemérita Universidad Autónoma de Puebla (BUAP) 1999, and M.Sc. and Ph.D. degrees in electrical engineering from Centro de Investigación y de Estudios Avanzados (CINVESTAV) of Instituto Politécnico Nacional, Mexico, 2000 and 2007, respectively. Currently works in BUAP as professor and researcher. Research interests: teletraffic analysis, cellular system dimensioning, performance modeling and evaluation of overlaid systems, packet networks and digital systems.

Gerardo Mino-Aguilar. Born in Mexico, 1971. Bachelor in Electronics Engineering at BUAP in 1995, master degree in power electronics at the Universidad de las Américas Puebla in 1999, and Ph.D. in electrical machines control at the Universitat Politècnica de Catalunya, Spain, 2006. Currently head of the graduate department on electronics engineering master's program, and electronics instrumentation at BUAP. Focused in digital systems, power electronics, power quality, power generation, electric drives and drivetrain electric vehicles.

Cortez Liliana. Born in Kiev, Ukraine in 1962. BS in Electrical Engineering and a Master of Sciences in power stations at the Polytechnical Institute of Kiev, Ukraine and a Ph.D in technical sciences at the Polytechnical State University of Saint Petersburg, Russia. Currently works as titular professor in the Faculty of Sciences in Electronics at BUAP, Mexico. Focused in digital systems, power electronics and power generation.

José Eligio Moisés Gutiérrez-Arias. Born in Puebla, Mexico, 1967. Bachelor of Mathematics and Master of Sciences in Mathematics in 1997 from the BUAP and PhD in Mathematical Sciences, same institution in 2003. Research: mathematical modeling and analysis of dynamic and digital systems. Currently he is researcher and professor at the Faculty of Electronic Sciences, BUAP.

José Fermi Guerrero-Castellanos. Born in Poza Rica, México. Bachelor degree in electronic science from BUAP, México in 2002, and M.Sc and Ph.D in automatic control from Grenoble Institute of Technology and Joseph Fourier University, Grenoble, France, in 2004 and 2008, respectively. Between January and June 2008, he was a Postdoctoral Researcher at GIPSALab Laboratory, Grenoble, France. Spent one year at the University Polytechnic of Puebla, Mexico as an assistant Professor, in June 2009 joined the Faculty of Electronics, at BUAP, Mexico, head of the Control and Power Systems Laboratory. Research interests: modeling, estimation and nonlinear control of cyber-physical systems. Applied concerns: Micro Unmanned Aerial Vehicles (MAVs ), wearable robots and digital systems.

Germán Ardul Muñóz-Hernández. BSc degree in electronic engineering from Veracruz Institute of Technology in 1988. In 1990, MSc degree in electronic systems from National Institute of Astrophysics Optic and Electronic (INAOE, Mexico). PhD degree from the University of Wales, Bangor in 2005. Worked at FAPATUX, a Pulp and Paper factory in Tuxtepec, Mexico, from 1986-1987 as programmer and from 1990-1993 in the area of Engineering and new projects. In 1993-1996, he was in the Electronics department at University of Puebla. He has been Visiting Engineer at South Carolina (Columbia, USA 1995), Michigan (Ann Arbor, USA 1996) and the CERN (Geneva, Switzerland 2007, 2009). Currently at BUAP. Areas of interest: digital systems, control systems applications and automation.
\end{abstract}

